\title{
The effect of poly (aspartic acid-co-lactic acid) nanospheres on the lung metastasis of B16BL6 melanoma cells by intravenous administration
}

\author{
KAORI HARA ${ }^{1}$, HIROYUKI TSUJIMOTO ${ }^{1}$, C.C. HUANG $^{2}$, YOSHIAKI KAWASHIMA ${ }^{3}$, \\ HARUKO MIMURA $^{4}$ and NOBUHIKO MIWA ${ }^{4}$
}

\author{
${ }^{1}$ Hosokawa Powder Technology Research Institute, 1-9 Shoudai, Tajika, Hirakata, Osaka 573-1132, Japan; \\ ${ }^{2}$ Hosokawa Micron Powder Systems, 10 Chatham Road, Summit, NJ 07901, USA; ${ }^{3}$ Aichi Gakuin University, \\ 1-100 Kusumoto, Chikusaku, Nagoya, Aichi 464-8650; ${ }^{4}$ Faculty of Pharmacy, School of BioSciences, \\ Prefectural University of Hiroshima, 562 Nanatsuka, Showbara, Hiroshima 727-0023, Japan
}

Received June 1, 2006; Accepted August 23, 2006

\begin{abstract}
Poly (aspartic acid-co-lactic acid) (PAL) has been investigated as a new biodegradable material for Drug Delivery Systems (DDS). Similar to the poly (lactic acid-coglycolic acid) (PLGA) nanospheres, the PAL nanospheres can control-release encapsulated drugs by hydrolysis and adhere to the mucous membranes to improve the drug absorption. In this study, the vitamin encapsulated PAL nanospheres were applied on mice to examine their effect on tumor metastasis and safety as an injectable DDS material for anti-cancer and other drugs. In the experiment, 6 C57BL/6 mice per group were intravenously administered with B16BL6 melanoma cells ( $1 \times 10^{5}$ per mouse $)$ and non-encapsulated PAL nanospheres or pro-vitamin encapsulated nanospheres respectively, while the control group was administered with B16BL6 cells alone. Two weeks later, the lungs of the mice were excised and metastatic foci on the lung surface were counted. The melanoma cell metastasis to lungs was prevented by intravenous co-injection of B16BL6 melanoma cells with $1.7 \mu \mathrm{g}$ of pro-vitamin E encapsulated PAL nanospheres. Its metastatic foci count (mean \pm SD) was $127 \pm 80$, which was better than the control $(246 \pm 95, \mathrm{p}<0.02)$. Also, applying the pro-vitamin $\mathrm{C}$ and pro-vitamin A encapsulated PAL nanospheres as well as the non-encapsulated PAL nanospheres slightly decreased the number of metastasis colonies in the lungs as compared to that of the control. These results suggested that PAL nanospheres did not promote the lung metastasis of B16BL6 melanoma cells.
\end{abstract}

Correspondence to: Dr Kaori Hara, Hosokawa Powder Technology Research Institute, 1-9 Shoudai, Tajika, Hirakata, Osaka 573-1132, Japan

E-mail: khara@hmc.hosokawa.com

Key words: poly (aspartic acid-co-lactic acid) nanosphere, poly (lactic acid-co-glycolic acid) nanosphere, metastasis, invasion, intravenous administration, DDS carrier
Thus, the PAL nanospheres are safe material for injection applications.

\section{Introduction}

Infiltration through basement membranes of cancer cells is essential for causing metastasis to other organs (1). When using a new carrier in the DDS for anti-cancer applications, for safety reasons, it is very important to assess the effect of the carrier on cancer metastasis. Poly (aspartic acid-co-lactic acid) (PAL) (2) is a bioabsorbable, biodegradable polymer developed over the last few years. The authors have optimized a process to efficiently encapsulate drugs in the sub-micron sized PAL nanospheres (3). These PAL nanospheres have about the same particle sizes as the poly (lactic acid-coglycolic acid) (PLGA) nanospheres $(4,5)$, which can improve the absorption of drugs and sustain their pharmacological effects. Because PAL nanospheres can control-release the drugs by hydrolysis similarly to the PLGA nanospheres, they are potential carriers of DDS for the transpulmonary (6-8) and transdermal $(9,10)$ drug administration. Furthermore, since the glass transition temperature of PAL nanospheres [aspartic acid:lactic acid $=17: 83$, weight-average molecular weight $(\mathrm{Mw})=9,000]$ is $\sim 60^{\circ} \mathrm{C}$, which is higher than the $45^{\circ} \mathrm{C}$ of PLGA nanospheres (lactic acid:glycolic acid $=$ 75:25, Mw $=20,000)$, PAL nanospheres are more advantageous in processing flexibility and storage stability; therefore, they can likely be applied in a wider range of applications. When designing new DDS with PAL nanospheres, it is important to assess the effect of these nanospheres on cancer metastasis at the development stage. Particularly, it was reported that the invasion and metastasis of cancer cells could be influenced by body conditions (11), such as blood hormones, and the routes of drug administration.

With an effort to adapt PAL nanospheres for transdermal applications, their safety of use was first investigated. Using the Boyden chamber method (12-14), which mimics human basement membranes, this study investigated the effect of a liquid mixture containing mouse melanoma B16BL6 cells 


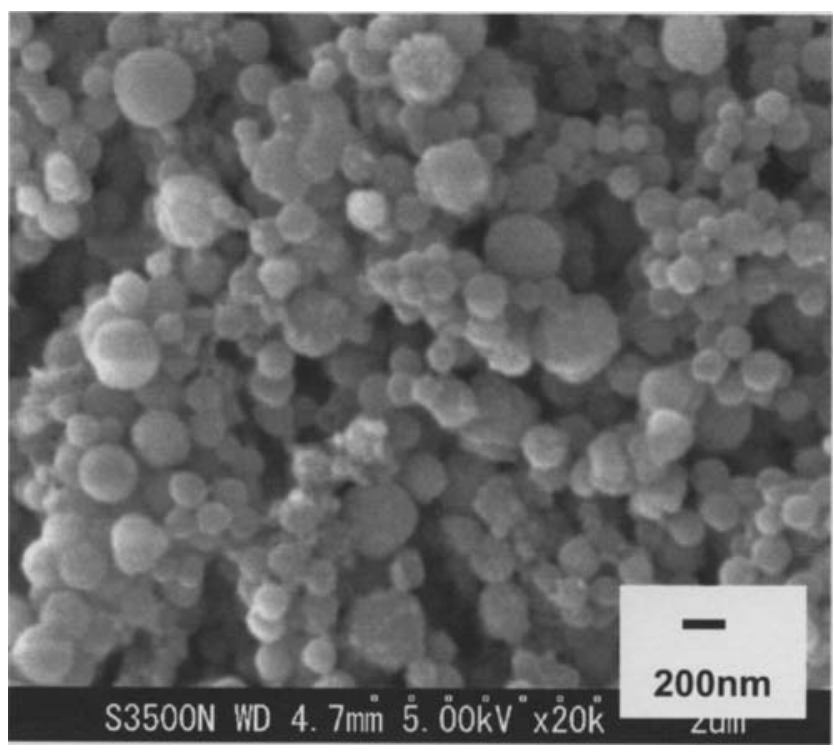

Figure 1. SEM photo of $8.6 \mathrm{wt} \%$ VC loaded PAL nanospheres.

and PAL nanospheres on the membrane invasion. It was found that the PAL nanospheres could inhibit melanoma membrane invasion (15). Cancer cells manage to infiltrate basement membranes by the following three steps: 1) thigmotaxis and adherence to the basement membranes, 2) production of matrix metalloprotease (MMP) precursors to dissolute the basement membranes, and 3) penetration of the basement membranes by means of cell deformation (16). It appeared that PAL nanospheres inhibited the activation of MMP precursors, because their free carboxyl radicals from the aspartic acid constituent had preferential bonding with the positively charged zinc ions, which were the active centers of MMP precursors. Furthermore, experimental results showed no promotion of the melanoma metastasis to other organs even though applying PAL nanospheres externally to the skin with a melanoma. Therefore, the PAL nanospheres are safe to use for transdermal drug delivery applications without the concern of cancer metastasis.

Taking one step further, this study was to assess the safety of using PAL nanospheres as a DDS carrier for anti-cancer agents and other drugs in the intravenous injection applications. The experiment was designed to investigate the effects of PAL nanospheres on cancer cell metastasis to the lungs when intravenously administering them together with mouse melanoma B16BL6 cells. The mouse melanoma B16BL6 cells were reported having a great tendency to metastasize to the lungs via the tail vein in a model study that created experimental lung metastatic tumors.

The experimental results suggested that intravenously administering PAL nanospheres were safe and did not promote the metastasis of melanoma cells to the lungs.

\section{Materials and methods}

Cell lines. The B16BL6 mouse melanoma cells (University of Texas M.D. Anderson Hospital, Houston, TX) were selected as the model cancer cells. The B16BL6 mouse melanoma cells have often been employed in the cancer metastasis

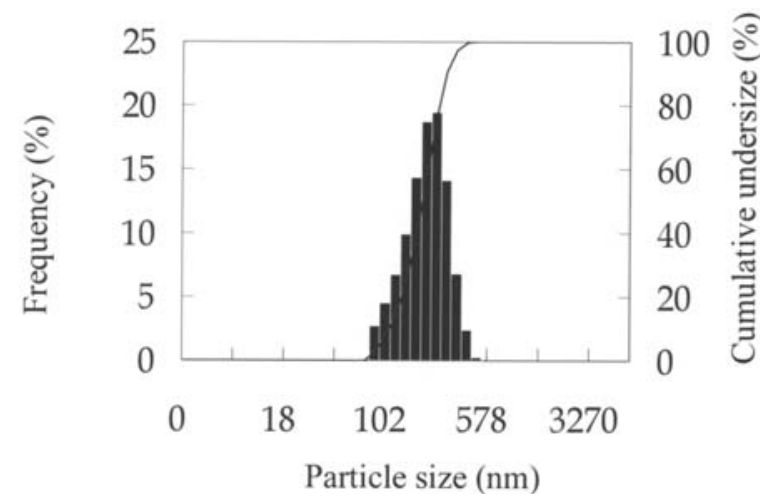

Figure 2. Particle size distribution of $8.6 \mathrm{wt} \%$ VC loaded PAL nanospheres.

experiments, because they quickly and selectively metastasize to the lungs after administration and proliferate in a short time.

Materials. PAL used in the experiment was the PAL15 made by Mitsui Chemicals, with an aspartic acid to lactic acid ratio of 17:83 and a weight-average Mw of 9,000. As a comparison, PLGA (PLGA7520; Wako Pure Chemical Industries) had a lactic acid to glycolic acid ratio of 75:25 with a weight-average Mw of 20,000. Model drugs encapsulated in the PAL nanospheres were: 1) Ascorbyl tetraisopalmitate (VC, Nikko Chemicals; a pro-vitamin C derivative), 2) DL- $\alpha$ tocopheryl acetate (VE, Eisai; a pro-vitamin E derivative), and 3) retinol palmitate (VA, Riken Vitamin; a pro-vitamin A derivative).

Polyvinyl alcohol (PVA, Kuraray; PVA403) was used as the dispersant for the production of PAL and PLGA nanospheres. In addition, negative control agent for melanoma lung cancer metastasis was ascorbyl tetraisopalmitate (VC); and, positive control agent for melanoma lung cancer metastasis was phorbol-12-myristate-13-acetate (PMA, Sigma Aldrich, Japan).

Preparation of PAL nanospheres. PAL nanospheres were prepared by the emulsion solvent diffusion (ESD) method, the same technique was used to prepare PLGA nanospheres (4,5). After dissolving $2 \mathrm{~g}$ of PAL in $20 \mathrm{ml}$ of acetone, $10 \mathrm{ml}$ of ethanol were added to make a polymer solution. The solution was then dropped into $50 \mathrm{ml}$ of $2 \%$ PVA aqueous solution at $40^{\circ} \mathrm{C}$ with a speed of $400 \mathrm{rpm}$. At this point, nano droplets were formed in the emulsion by the selfemulsification at the droplet interface due to rapid diffusion and counter diffusion of the solvents. After evaporating the organic solvents and removing excess PVA by washing and centrifugation, the remainder was freeze-dried to obtain a dry PAL nanosphere powder (i.e. sample group iv). With the same procedure, vitamin encapsulated PAL nanospheres could be produced by adding $0.2 \mathrm{~g}$ of $\mathrm{VC}, \mathrm{VE}$, or VA separately in the above polymer solution.

The encapsulation ratio of $\mathrm{VC}, \mathrm{VE}$, and $\mathrm{VA}$ in the encapsulated PAL nanospheres was 8.6, 11.0, and 7.5\% representing the sample group i, ii, and iii, respectively. After freeze-drying, the nanosphere particles were re-dispersed in the purified water for particle size measurement by the dynamic light-scattering method. All particles were spherical 


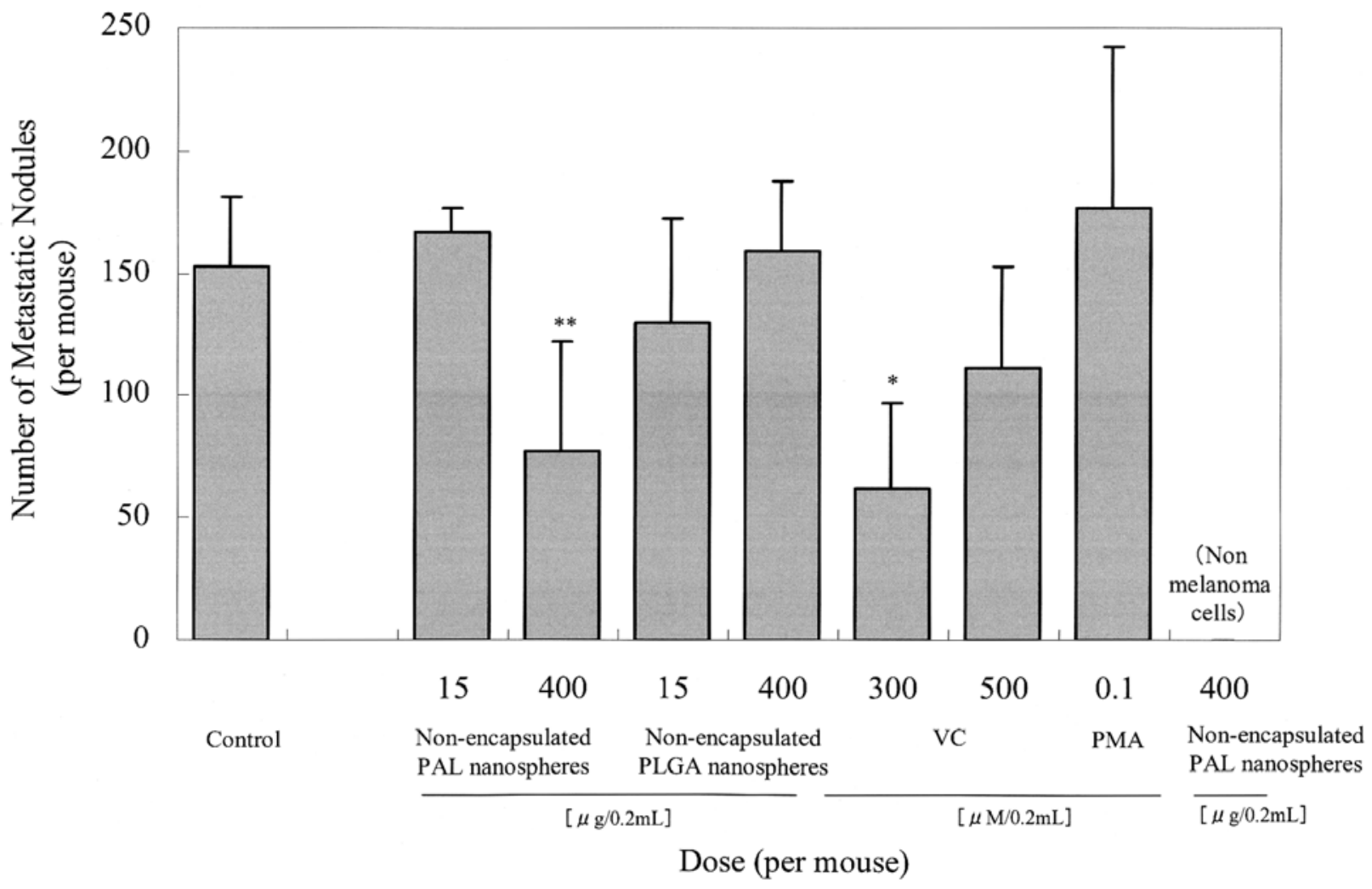

Figure 3 . The number of metastatic nodules on the lung intravenously administered with VC, PMA and non-encapsulated nanospheres $\left({ }^{*} \mathrm{p}<0.01,{ }^{* *} \mathrm{p}<0.05\right)$.

shape and had an average particle size of $200 \mathrm{~nm}$ with narrow particle size distribution. Fig. 1 shows the scanning electron microscope (SEM) photos of VC loaded PAL nanospheres, and Fig. 2 shows its particle size distribution. In addition, a control sample of non-encapsulated PLGA nanospheres having an average particle size of $260 \mathrm{~nm}$ (i.e. sample group v) was also prepared for the experiment to assess the influence of different carrier materials on the test results.

Cancer metastasis assay (17). The following describes the procedures used to study the possibility of cancer metastasis stimulated by a single dose of PAL nanospheres. 1) Trypsintreated B16BL6 mouse skin melanoma cells (MEM/10\% FCS, $1 \times 10^{5}$ cells) in their logarithmic growth phase were incubated with the nanospheres (from groups i to v), negative control agent, or positive control agent separately in a rotator for $1 \mathrm{~h}$ at $37^{\circ} \mathrm{C}$. 2) The test solution prepared in 1) was injected into $\mathrm{C} 57 \mathrm{BL} / 6$ mice (eight-week-old male, $\mathrm{n}=6$ to $8 /$ test group) via its tail vein. 3) The mice were terminated 11 or 14 days after inoculation with the melanoma cells. Their lungs were excised, rinsed and fixed in the Bouins solution. The metastatic foci on the lung surfaces were counted (mean \pm SD) under a stereomicroscope. The metastatic foci count was then compared with that of the control group, which was not given test substance.

The doses of the non-encapsulated PAL nanospheres (group iv) and the non-encapsulated PLGA nanospheres control (group v) applied in the experiment were based on the commercial leupline ${ }^{\mathrm{TM}}$ for injection (Takeda Chemical
Industries, Ltd.: packaging insertion of leuplin for injection $1.88,3.75$; kit 1.88 and kit 3.75 ), which contains PLGA microspheres to treat cancers such as prostate gland and postmenopausal breast cancer. Specifically, a single dose of leupline was $33.75 \mathrm{mg}$ of PLGA per $60 \mathrm{~kg}$ of patient. Taking the proportion for an average weight of the C57BL/6 mouse ( $20 \mathrm{~g}$ at eight weeks old), it came to $11.25 \mu \mathrm{g}$ of PLGA per $20 \mathrm{~g}$. In the experiment, the dose was set at $15 \mu \mathrm{g} / 20 \mathrm{~g}$ to positively confirm the safety of the nanospheres. In addition, a dose as high as $400 \mu \mathrm{g} / 20 \mathrm{~g}$ ( $>25$ times the standard dose) was also tested.

The doses of the vitamin derivative encapsulated PAL nanospheres (from groups i to iii) were based on the commercially available functional beauty essences (10) containing PLGA nanospheres, whose safety was previously confirmed. Specifically, by determining the concentration of vitamin derivatives in the beauty essence and the dose of the non-encapsulated PAL nanospheres, the required amount of vitamin derivatives in the PAL nanospheres could be calculated.

\section{Results and Discussion}

Fig. 3 shows the number of metastatic cancer nodules in the lungs measured 11 days after intravenously administering melanoma cells without any nanospheres (control group), and with non-encapsulated PAL nanospheres (group iv) as well as non-encapsulated PLGA nanospheres (group v). Fig. 4 shows representative lung tissue samples from each test group. 

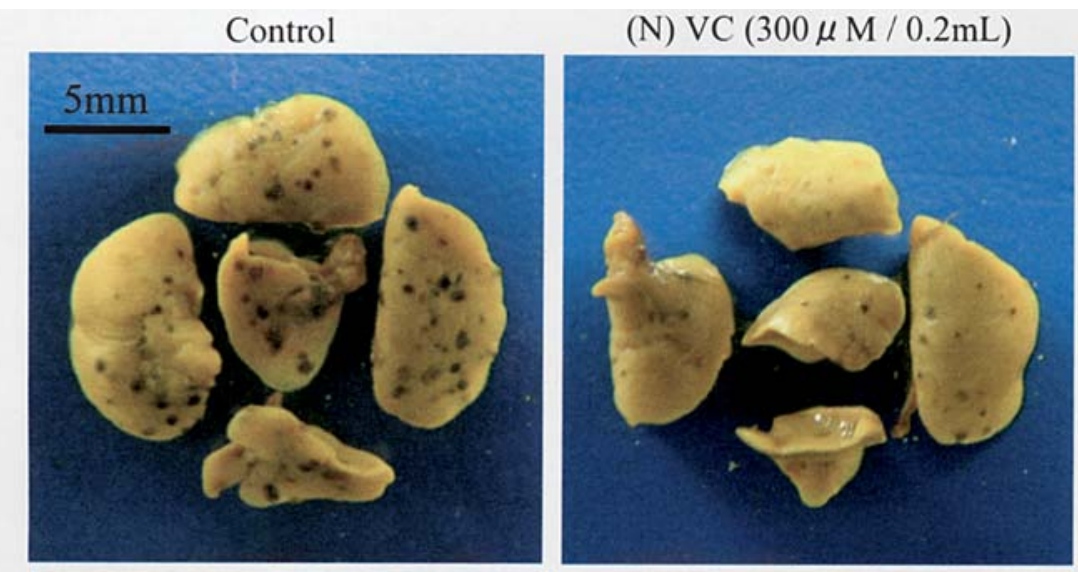

(P) $\operatorname{PMA}(0.1 \mu \mathrm{M} / 0.2 \mathrm{~mL})$

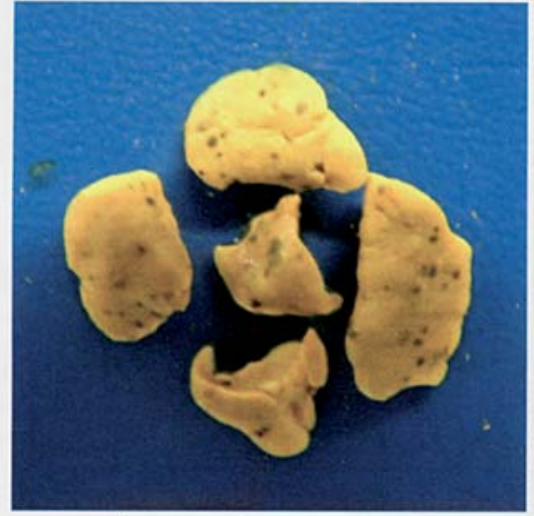

(iv) Non-encapsulated PAL nanospheres $(400 \mu \mathrm{g} / 0.2 \mathrm{~mL})$

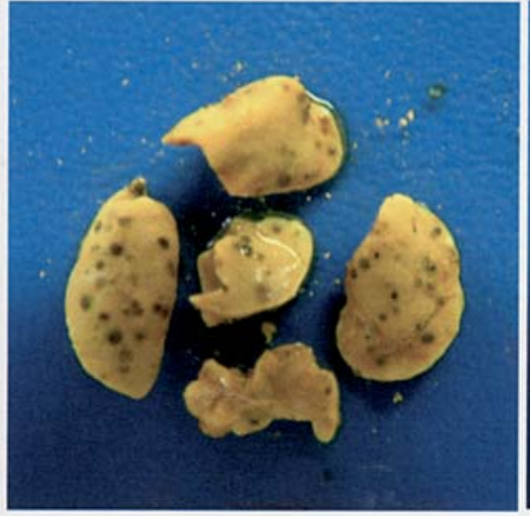

( v ) Non-encapsulated PLGA nanospheres $(15 \mu \mathrm{g} / 0.2 \mathrm{~mL})$
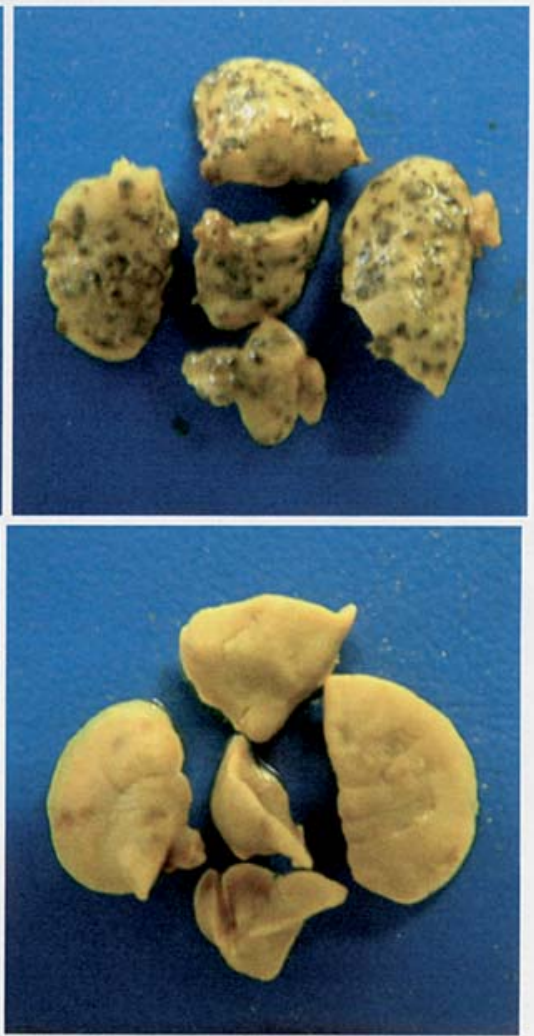

Non-encapsulated PAL nanospheres $(400 \mu \mathrm{g} / 0.2 \mathrm{~mL})$

(Non B16BL6 melanoma cells)

Figure 4. Representative samples of the C57BL/6 mouse lung intravenously administered with VC, PMA and non-encapsulated nanospheres.

Effect of administering negative control and positive control. To validate the in vitro assay, two additional groups were tested. They were the negative control group (group $\mathrm{N}$ ) administered with VC, which is known to suppress melanoma metastasis to the lungs (18); and, the positive control group (group P) administered with phorbol-12-myristate-13acetate (PMA), which is a powerful cancer promoter (19). The evaluation procedures of the groups $\mathrm{N}$ and $\mathrm{P}$ were the same as those mentioned above in the cancer metastasis assay section. The VC dosages applied herein were the same as the dosages of Asc2P (ascorbic acid-2-O-phospate Mg salt) and Asc2G (ascorbic acid-2-O- $\alpha-\mathrm{D}$-glycoside) used in our previous evaluation system, which were proven effective to repress melanoma invasion (1). Also, PMA dosages were set at the same level as those used in the chemo-invasion assay (19), which showed positive invasion of human hepatocellular carcinoma (HCC), SNU-387 and SNU-398.

The number of cancer nodules in the control group was $153 \pm 28$ (mean $\pm \mathrm{SD}$ ). Cancer cell colonies were found on the surfaces of five lung lobules as grains colored black by melanin pigment. When intravenously administering $\mathrm{VC}$ to group $\mathrm{N}$ with melanoma cells, the number of cancer nodules was reduced to $62 \pm 35$ and $112 \pm 41$ with $\mathrm{p}<0.01$ at the concentrations of 300 and $500 \mu \mathrm{M}$, respectively.

It is believed that the VC taken by the melanoma cells during incubation in the rotator could suppress cancer invasion. It appeared that the reduced vitamin $\mathrm{C}$ converted from $\mathrm{VC}$ by esterase eliminated the reactive oxygen species and free radicals in the melanoma cells. This, in turn, decreased the cancer cell mobility and thigmotaxis, and limited the production of the MMP-2 and MMP-9 needed for cancer invasion (1).

When intravenously administering PMA to group P with melanoma cells, the number of cancer nodules found was $177 \pm 65$ (mean $\pm \mathrm{SD})$ at concentrations of $0.1 \mu \mathrm{M}$. It seemed that the cancer cell invasion to the lung tissue was stimulated by the carcinogenic promotion of PMA when it was incubated with melanoma cells in the rotator. It is believed that the PMA activated the protein kinase $\mathrm{C}$ (PKC), a signal transferring material in a cancer cell, to cause the production of MMP-9. The MMP-9, in turn, promoted cancer metastasis and resulted in a marked increase of cancer nodules in the lungs.

Therefore, this method of assaying by intravenously injecting each agent and melanoma cells at the same time is an appropriate method for assaying the capability of melanoma cells to metastasize to the lungs.

Effect of administering non-encapsulated PAL and PLGA nanospheres. The number of lung cancer nodules (mean $\pm \mathrm{SD}$ ) from the test group receiving non-encapsulated PAL nanospheres along with melanoma cells was $167 \pm 9.3$ with a dose of $15 \mu \mathrm{g} /$ mouse, and $76 \pm 45(\mathrm{p}<0.05)$ with a dose of $400 \mu \mathrm{g} /$ mouse. 


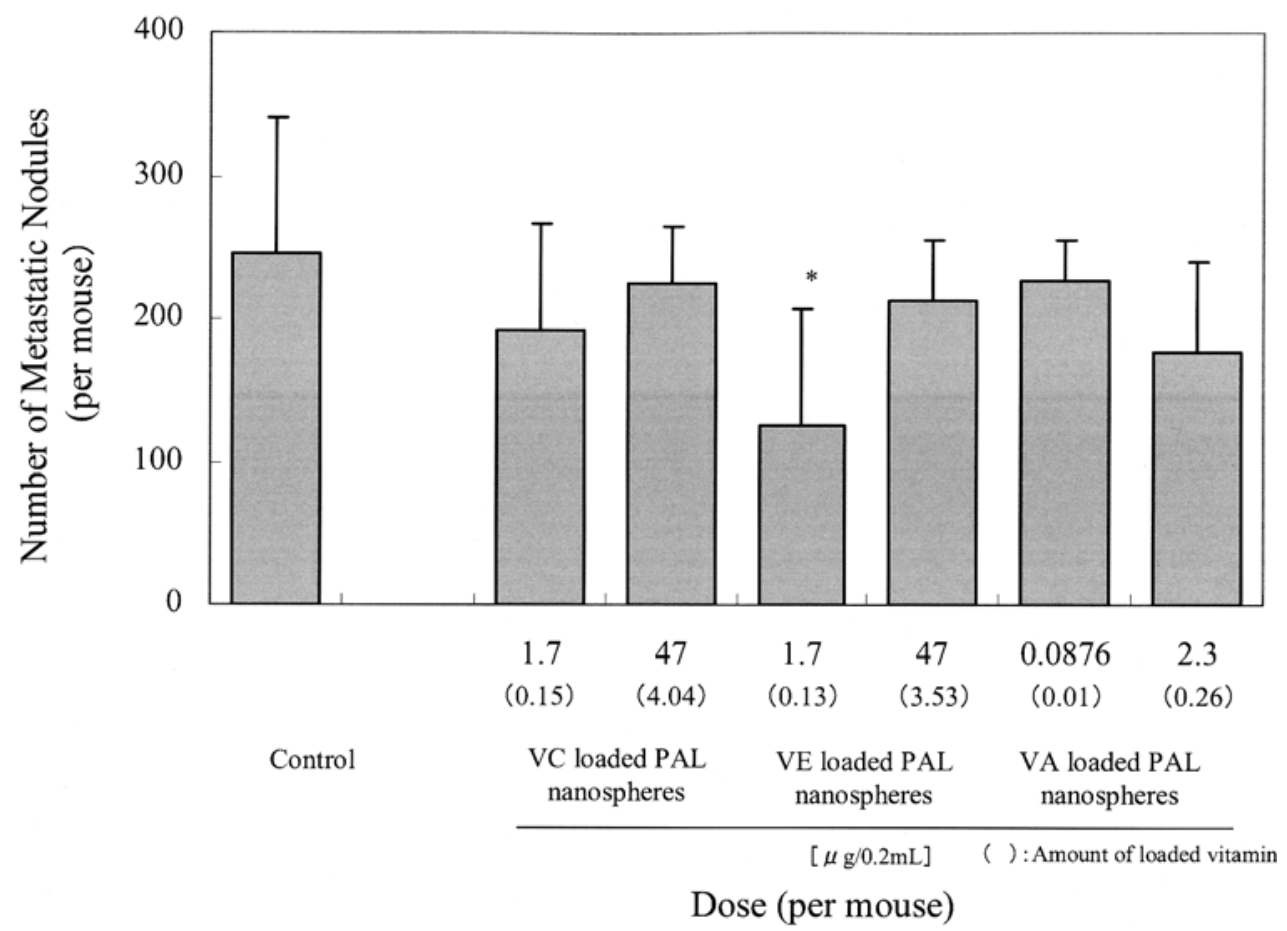

Figure 5. The number of metastatic nodules on the lung intravenously administered with VC, VE, and VA loaded PAL nanospheres ( $\left.{ }^{*} \mathrm{p}<0.02\right)$.

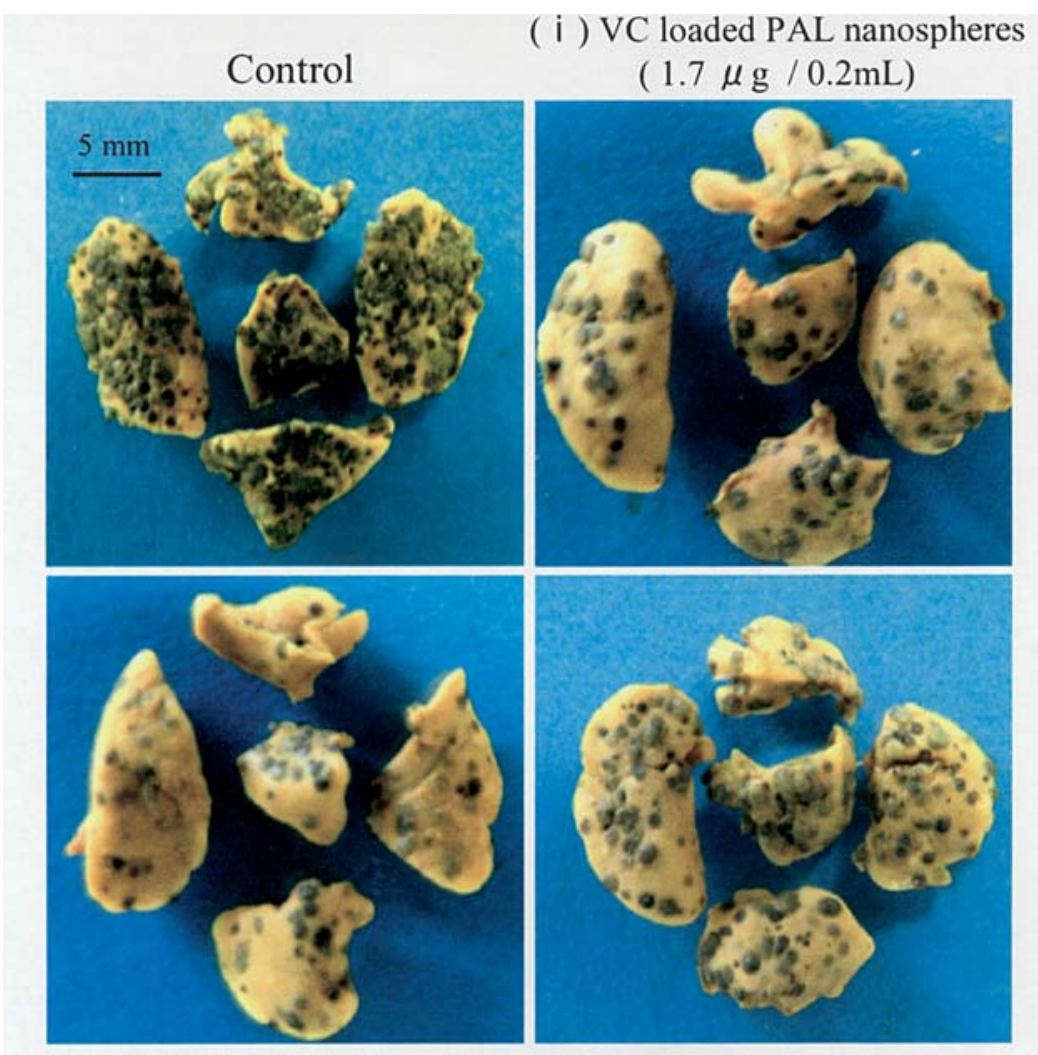

(ii ) VE loaded PAL nanospheres (iii) VA loaded PAL nanospheres $(1.7 \mu \mathrm{g} / 0.2 \mathrm{~mL})$ $(2.3 \mu \mathrm{g} / 0.2 \mathrm{~mL})$

Figure 6. Representative samples of the C57BL/6 mouse lung intravenously administered with VC, VE, and VA loaded PAL nanospheres.

Similarly, in the non-encapsulated PLGA nanosphere group, we discerned no significant difference in the cancer nodule count from the control group regardless of the particle size or concentration administered. The PLGA nanospheres were proven safe for hypodermic injection. This indicated that PAL nanosphere would have no adverse effect 
on the cancer metastasis and it is safe to use as a DDS carrier.

Furthermore, non-encapsulated PAL nanospheres without melanoma cells were intravenously administered to the mouse. There was no observation of cancer cells in the excised lungs $(n=1)$. This result indicated that the lung cancer found in this assay was solely caused by the intravenous administration of melanoma cells. The PAL nanospheres themselves are not carcinogenic.

The effects of vitamin derivative encapsulated PAL nanospheres. Fig. 5 shows the number of metastasized lung cancer nodules found in the control group and in the groups given vitamin derivative encapsulated PAL nanospheres mixed with melanoma cells (from groups i to iii) 14 days after intravenously administering the preparations. Fig. 6 shows representative lung tissues from each group. While the control group had cancer nodules at $246 \pm 95$, those from the groups with VC, VE, and VA encapsulated PAL nanospheres were: from low to high doses, $193 \pm 74$ (group i -1 ), $225 \pm 39$ (group i -2), 127 \pm 80 (group ii -1), 213 \pm 42 (group ii -2), $226 \pm 30$ (group iii -1) and $177 \pm 63$ (group iii -2). It appeared that there was no dependence on the dose size. Except group ii -1 , there was no significant difference between the control group and other test groups. This result confirms that vitamin derivative encapsulated PAL nanospheres did not have adverse effect on the cancer cell metastasis. The absence of metastasis suppression effect with the VC encapsulated PAL nanospheres might be attributed to its extremely small concentration $(\sim 1 / 80-1 / 2,300$ of that used in the negative control, which showed significant metastasis suppression). In group ii -1 where the mice were each given $1.7 \mu \mathrm{g}$ of VE encapsulated PAL nanospheres, there was metastasis suppression by $51 \%$ in comparison to the control group. It is believed that the free carboxyl radicals in the PAL inhibited the activation of MMP needed for metastasis; and, the VE derivative eliminated the weak reactive oxygen species in cells, which could trigger the melanoma metastasis.

In conclusion, to assess the safety of using PAL nanospheres, a biocompatible, bioabsorbable polymer, as a DDS carrier for intravenous applications, was applied to B16BL6 mouse melanoma cells to investigate their effect on the cancer cell metastasis in a mouse model. 1) It was found that both non-encapsulated PAL nanospheres and vitamin derivative encapsulated PAL nanospheres exhibited no adverse effect on the cancer cell metastasis. 2) Vitamin E encapsulated PAL nanospheres exhibited $\sim 50 \%$ suppression effect on the cancer cell metastasis in comparison to the control group.

Results obtained within the scope of this study suggested that the PAL nanospheres are safe to use for intravenous injection from the perspective of cancer cell metastasis.

\section{References}

1. Miwa N: Bio Kousankazai Purovitamin C. Miwa N (ed). Fragrance Journal, Tokyo, pp37-92, 1999.

2. Shinoda H, Asou Y, Suetsugu A and Tanaka K: Synthesis and Characterization of amphiphilic biodegradable copolymer, poly (aspartic acid-co-lactic acid). Macromol Biosci 3: 34-43, 2003.
3. Tsujimoto $\mathrm{H}$ and Hara K: Development of functional cosmetics using vitamin derivatives loaded biodegradable polymeric nanospheres. Pharm Tech Japan 21: 53-64, 2005.

4. Kawashima Y, Okumura M and Takenaka H: Spherical crystallization: direct spherical agglomeration of salicylic acid crystals during crystallization. Science 216: 1127-1128, 1982.

5. Kawashima Y, Yamamoto H, Takeuchi H, Hino T and Niwa T: Properties of a peptide containing DL-lactide/glycolide copolymer nanospheres prepared by novel emulsion diffusion methods. Eur J Pharmaceutics Biopharm 45: 41-48, 1998.

6. Yamamoto H, Hoshina W, Kurashima H, Takeuchi H, Kawashima Y, Yokoyama T and Tsujimoto H: Engineering of poly (lactic-co-glycolic acid) nano-composite particle for dry powder inhalation dosage forms of insulin with spray fluidized bed granulating system. J Soc Powder Technol 41: 514-521, 2004 .

7. Yamamoto H, Kurashima H, Katagiri D, Yang M, Takeuchi H, Kawashima Y, Yokoyama T and Tsujimoto H: Poly (lactic-coglycolic acid) nanosphere composite prepared with mecahnofusion dry powder composition system for improving pulmonary insulin delivery with dry powder inhalation. Yakuzaigaku 64 : 245-253, 2004.

8. Tsujimoto H, Hara K and Kawashima Y: Evaluation of glycaemia control in beagle dogs by the administration of insulin encapsulated PLGA nano-composite preparations. J Soc Powder Techonol 42: 765-772, 2005.

9. Tsujimoto H, Hara K, Huang CC, Yokoyama T, Yamamaoto H, Takeuchi H, Kawashima Y, Akagi K and Miwa N: Percutaneous absorption study of biodegradable PLGA nano-spheres via human skin biopsies. J Soc Powder Technol 416: 867-875, 2004.

10. Hosokawa Micron cosmetics, Nanocrysphere, www. nanocrysphere.com

11. Murata J, Ayukawa K, Ogasawara M, Watanabe H and Saiki I: Induction of autocrine factor inhibiting cell motility from murine B16-BL6 melanoma cells by $\alpha$-melanocyte stimulating hormone. Int J Cancer 80: 889-895, 1999.

12. Nakayama T, Matsubara T, Makiyama T, Nagao N, Kageyama K, Saiki I, Iwagaki H, Nakamura T and Miwa N: Establishment and application of tumor invasion model as a system for screening tumor metastasis inhibitors: inhibitory effects of ascorbic acid-2phosphate on tumor invasion and its molecular mechanism. Final Reports for Research Grants for Meat and Meat Products 13: 387-395, 1994.

13. Jian-Wen L, Nagao N, Kageyama K and Miwa N: Antimetastatic effect of an autooxidation-resistant and lipophilic ascorbic acid derivative through inhibition of tumor invasion. Anticancer Res 20: 113-118, 2000.

14. Nagao N, Nakayama T, Etoh T, Saiki I and Miwa N: Tumor invasion is inhibited by phosphorylated ascorbate via enrichment of intracellular vitamin $\mathrm{C}$ and decreasing of oxidative stress. Cancer Res Clion Oncol 126: 511-518, 2000.

15. Tsujimoto $\mathrm{H}$, Hara $\mathrm{K}$, Mimura $\mathrm{H}$ and Miwa $\mathrm{N}$ : Inhibitory Effects of poly (aspartic acid-co-lactic acid) nanospheres on tumor invasion through the reconstituted basement membrane. $\mathrm{J}$ Soc Powder Technol 43: 86-91, 2006.

16. Miyazaki K and Higashi M: Matorikkusu metaro purotea-ze no kouzou to kinou - toku ni gan no sinjun - teni ni okeru yakuwari. (In Japanese) Biochemistry 68: 1791-1807, 1996.

17. Jian-Wen L, Nagao N, Kageyama N and Miwa N: Antimetastatic and anti-invasive ability of phospho-ascorbyl palmitate through intracellular ascorbate enrichment and the resultant antioxidant action. Oncol Res 11: 479-487, 1999.

18. Miwa N: Rejuvenation and Skin Protection, and Biotechonology. CMC Publishing, Tokyo, pp341-350, 2003.

19. Nasun H and Lee S-T: An absolute role of the PKC-dependent NF- $\kappa \mathrm{B}$ activation for induction of MMP-9 in hepatocellular carcinoma cells. Biochem Biophys Res Commun 305: 428-433, 2003. 